\title{
Direct Evidence for Wake-Related Increases and Sleep-Related Decreases in Synaptic Strength in Rodent Cortex
}

\author{
Zhong-Wu Liu, ${ }^{1,2}$ Ugo Faraguna, ${ }^{3}$ Chiara Cirelli, ${ }^{3}$ Giulio Tononi, ${ }^{3}$ and Xiao-Bing Gao ${ }^{1}$ \\ ${ }^{1}$ Department of Obstetrics, Gynecology, and Reproductive Sciences, Yale University School of Medicine, New Haven, Connecticut 06510, ${ }^{2}$ Department of \\ Neurobiology, Yunyang Medical College, Yunyang 442000, Hubei, China, and ${ }^{3}$ Department of Psychiatry, University of Wisconsin-Madison, Madison, \\ Wisconsin 53719
}

Despite evidence that waking is associated with net synaptic potentiation and sleep with depression, direct proof for changes in synaptic currents is lacking in large brain areas such as the cerebral cortex. By recording miniature EPSCs (mEPSCs) from frontal cortex slices of mice and rats that had been awake or asleep, we found that the frequency and amplitude of mEPSCs increased after waking and decreased after sleep, independent of time of day. Recovery sleep after deprivation also decreased mEPSCs, suggesting that sleep favors synaptic homeostasis. Since stronger synapses require more energy, space, and supplies, a generalized renormalization of synapses may be an important function of sleep.

\section{Introduction}

The functions of sleep are still unknown (Cirelli and Tononi, 2008; Siegel, 2009), but it is now clear that some molecular and cellular processes in the brain change in close temporal relationship with the sleep/wake cycle. Specifically, the expression of genes and the synthesis of proteins involved in synaptic transmission fluctuate with the sleep/wake cycle, suggesting that synaptic efficacy may undergo homeostatic changes driven by behavioral state (Cirelli et al., 2004; Cirelli, 2009). In line with this idea, a recent in vivo study in rats measured molecular and electrophysiological markers of synaptic strength after periods of sleep and wakefulness (Vyazovskiy et al., 2008). At the molecular level, the best established mechanism for the expression of long-term potentiation and long-term depression involves the trafficking of postsynaptic glutamatergic AMPA receptors containing the glutamate receptor (GluR1)-1 subunit. The delivery of these receptors to excitatory synapses is associated with increases in synaptic strength, while their removal from synapses is associated with synaptic depression (Collingridge et al., 2004; Malenka and Bear, 2004). It was found that in rat cortical and hippocampal synaptoneurosomes, a preparation that enriches for synaptic proteins, GluR1-containing AMPA receptor levels were $~ 40 \%$ higher in wakefulness than in sleep (Vyazovskiy et al., 2008). Phosphorylation changes of AMPA receptors, and of the enzymes CamKII and GSK $3 \beta$, were also consistent with net synaptic potentiation during wakefulness and depression during sleep (Vyazovskiy et al., 2008). Electrophysiologically, it was shown using cortical

Received March 18, 2010; revised April 21, 2010; accepted May 17, 2010.

This work was supported by National Institutes of Health (NIH) Grant DK 070723 (X.-B.G.) and NIH Director's Pioneer Award (G.T.).

Correspondence should be addressed to either of the following: Xiao-Bing Gao, Department of OB/GYN, Yale University School of Medicine, 333 Cedar Street, New Haven, CT 06520, E-mail xiao-bing.gao@yale.edu; or Giulio Tononi, Department of Psychiatry, University of Wisconsin-Madison, 6001 Research Park Boulevard, Madison, WI 53719. E-mail gtononi@wisc.edu.

DOI:10.1523/JNEUROSCI.1409-10.2010

Copyright $\odot 2010$ the authors $\quad 0270-6474 / 10 / 308671-05 \$ 15.00 / 0$ electrical stimulation and local field potential recordings that both the slope and amplitude of cortical evoked responses, classic in vivo measures of synaptic strength, also increased after wakefulness and decreased after sleep (Vyazovskiy et al., 2008). Finally, a recent unit recording study indicates that mean firing rate in the cerebral cortex increases after periods of wakefulness and decreases after periods of sleep, consistent with a net change in synaptic strength (Vyazovskiy et al., 2009).

A net increase in cortical synaptic strength with waking would pose a significant burden in terms of energy, space, cellular supplies, and memory saturation (Attwell and Laughlin, 2001). If sleep did renormalize synaptic strength, it would be highly beneficial and could account at least in part for its universality (Tononi and Cirelli, 2006; Cirelli and Tononi, 2008). However, molecular changes do not necessarily reflect functional changes, and changes in field potentials, as well as changes in mean firing rates, could be influenced by factors other than synaptic strength. To obtain a direct measure of cortical synaptic strength after wake and sleep, it would be important to record miniature EPSCs (mEPSCs) from brain slices of animals that have been awake or asleep, and examine changes in their frequency and amplitude. Here, we set out to examine whether a net increase in synaptic strength after wake might occur in the cerebral cortex, the part of the brain that has been most closely linked to sleep function (Moruzzi, 1966; Horne, 1988; Tononi and Cirelli, 2006; Krueger et al., 2008). Moreover, we asked whether an increase in synaptic strength after wake would be counteracted by sleep, consistent with a homeostatic process. Specifically, we examined the effects of sleep and wake on mEPSCs of cortical neurons located in layers II/III of the rat and mouse frontal cortex.

\section{Materials and Methods}

Sleep deprivation. Male mice (C57BL/6, 3-4 weeks old, Jackson Laboratory) or rats (Sprague Dawley, 4-8 weeks old, Charles River, Laboratories) were housed individually and maintained on a $12 \mathrm{~h}$ light/12 h dark cycle (lights on at 6 A.M.) with food and water available ad libitum. For 
each of the four experiments, animals were divided into two groups (controls and spontaneously awake or sleep deprived) and the experiment was performed in the home cages. Sleep deprivation (SD) was performed as reported previously (Rao et al., 2007). SD animals were sleep deprived for $4 \mathrm{~h}$ (8:00 A.M. to 12:00 P.M. by gentle handling, while controls were allowed to sleep undisturbed from 8:00 A.M. to 12:00 P.M.) Controls and spontaneously awake rats were monitored using video camera and/or visual observation, and only rats that were asleep or awake for most (at least $65 \%$ ) of the previous $4 \mathrm{~h}$ were selected for the experiments. In some rats recovery sleep (RS) was allowed for $2 \mathrm{~h}$ (from 12:00 P.M. to 2:00 P.M.) following SD. RS was monitored by using standard electroencephalogram (EEG) recordings, as in previous reports (Vyazovskiy et al., 2008). Sleep stages were scored off-line by visual inspection of $4 \mathrm{~s}$ epochs (SleepSign, Kissei). All animals were habituated to the presence of the experimenter and gentle handling for $3 \mathrm{~d}$ before the SD experiment. After the experiments, all animals were killed for electrophysiological studies. All animal procedures followed the National Institutes of Health Guide for the Care and Use of Laboratory Animals, and facilities were reviewed and approved by the Institutional Animal Care and Use Committee of Yale University School of Medicine.

Electrophysiology. Coronal cortical slices, $300 \mu \mathrm{m}$ thick, were prepared from frontal cortex (Bregma, $\geq 3 \mathrm{~mm}$; lateral, $0.5-1.5 \mathrm{~mm}$ ) of mice and rats as described previously (Rao et al., 2007). Briefly, mice or rats were anesthetized with ether and then decapitated. Brains were rapidly removed and immersed in an oxygenated bath solution at $4^{\circ} \mathrm{C}$ containing the following (in mM): 220 sucrose, $2.5 \mathrm{KCl}, 1 \mathrm{CaCl}_{2}, 6 \mathrm{MgCl}_{2}, 1.25$ $\mathrm{NaH}_{2} \mathrm{PO}_{4}, 26 \mathrm{NaHCO}_{3}$, and 10 glucose, pH 7.3, with $\mathrm{NaOH}$. After preparation, slices were maintained in a holding chamber with artificial CSF (bubbled with $5 \% \mathrm{CO}_{2}$ and $95 \% \mathrm{O}_{2}$ ) containing the following (in $\mathrm{mm}$ ): $124 \mathrm{NaCl}, 3 \mathrm{KCl}, 2 \mathrm{CaCl}_{2}, 2 \mathrm{MgCl}_{2}, 1.23 \mathrm{NaH}_{2} \mathrm{PO}_{4}, 26 \mathrm{NaHCO}_{3}$, and 10 glucose, $\mathrm{pH}$ 7.4, with $\mathrm{NaOH}$. Slices were transferred to a recording chamber constantly perfused with a bath solution $\left(33^{\circ} \mathrm{C}\right)$ at $2 \mathrm{ml} / \mathrm{min}$ after at least $1 \mathrm{~h}$ of recovery.

Whole-cell voltage clamp (at $-60 \mathrm{mV}$ ) was performed to observe miniature postsynaptic currents in layer II/III cortical neurons with a Multiclamp 700A amplifier (Molecular Devices). The patch pipettes (tip resistance, 4-6 M $\Omega$ ) were made of borosilicate glass (World Precision Instruments) with a pipette puller (Sutter P-97) and backfilled with a pipette solution containing the following (in $\mathrm{mM}$ ): $135 \mathrm{~K}$-gluconate (or Cs-gluconate), $2 \mathrm{MgCl}_{2}, 10 \mathrm{HEPES}, 1.1 \mathrm{EGTA}, 2 \mathrm{Mg}$-ATP, $10 \mathrm{Na}_{2}$ phosphocreatine, and $0.3 \mathrm{Na}_{2}-\mathrm{GTP}, \mathrm{pH} 7.3$, with $\mathrm{KOH}$. Miniature EPSCs were recorded under voltage clamp at $-60 \mathrm{mV}$ in the presence of tetrodotoxin $(0.5 \mu \mathrm{M})$ and a $\mathrm{GABA}_{\mathrm{A}}$ receptor antagonist, picrotoxin (50 $\mu \mathrm{M})$. Both input resistance and series resistance were monitored throughout the experiments; the series resistance (between 20 and 40 $\mathrm{M} \Omega$ ) was partially compensated for by the amplifier. Only recordings with stable series resistance and input resistance throughout the experiment were accepted. All data were sampled at $3-10 \mathrm{kHz}$ and filtered at $1-3 \mathrm{kHz}$ with an Apple Macintosh computer using Axograph X (AxoGraph Scientific). mEPSC events were detected and analyzed with AxoGraph X and plotted with Igor Pro software (WaveMetrics) as described previously (Rao et al., 2007).

\section{Results}

Our experiments focused on the frontal cortex because it is the site where molecular and electrophysiological indices of synaptic strength were found to change after sleep and wake in a previous study (Vyazovskiy et al., 2008). Moreover, the frontal cortex shows the most conspicuous homeostatic changes in sleep slow-wave activity, increasing after wake and decreasing after sleep, and new evidence indicates that the amount of slowwave activity may also be an indirect indicator of net synaptic strength (Vyazovskiy et al., 2008).

In the first experiment, we harvested coronal cortical slices $(300 \mu \mathrm{m})$ from the frontal cortex of rats that had been mainly spontaneously awake during the first part of the dark phase $(n=$ 4; killed around 12:00 A.M.) or mainly asleep for several hours during the light phase ( $n=4$; killed around 5:00 P.M.). mEPSCs were recorded in layer II/III pyramidal neurons, and detection and analysis of mEPSC frequency and amplitude were performed as described previously (Rao et al., 2007). Changes in mEPSC frequency are thought to result from modification of the presynaptic component of synaptic transmission, while amplitude changes indicate alterations in the postsynaptic component (Ungless et al., 2001). We found that the frequency of mEPSCs was significantly higher in awake rats $(3.10 \pm 0.5 \mathrm{~Hz}, n=25$ cells $)$ than in sleeping rats $(1.69 \pm 0.21 \mathrm{~Hz}, n=24$ cells; $p<0.05, t$ test $)$ (Fig. $1 A$ ). The mean amplitude of mEPSCs was not significantly different between these two groups (sleeping group: $24.4 \pm 1.4$ pA, $n=24$; wake group: $25.4 \pm 1.3 \mathrm{pA}, n=25$ ); however, the cumulative probability curves for the amplitude of mEPSC events recorded from wake rats shifted significantly to the right compared with those recorded in the sleeping group $(p<0.01$, Kolmogorov-Smirnov test) (Fig. $1 A$ ). These results suggest that changes in behavioral states can affect synaptic strength in layer II/III pyramidal neurons of the frontal cortex, resulting in a net increase after prolonged wake and a net decrease after sleep. However, sleeping and spontaneously awake rats were killed at different times of day, leaving open the possibility that circadian time, rather than (or in addition to) behavioral state, could account for the observed changes.

To tease apart the effects of sleep/wake from those of circadian time, we used two groups of rats killed at the same time of day, the first group after $4 \mathrm{~h}$ of sleep (controls, $n=7$ ) and the second group after $4 \mathrm{~h}$ of SD by gentle handling (from 8:00 A.M. to 12:00 P.M., $n=7$ ). As before, we measured the synaptic efficacy of layer II/III pyramidal neurons in the frontal cortex. We found that the frequency of mEPSCs was significantly higher in rats kept awake for $4 \mathrm{~h}$ than in controls allowed to sleep (SD rats: $5.71 \pm 1.09 \mathrm{~Hz}$, $n=29$ cells; controls: $2.31 \pm 0.40 \mathrm{~Hz}, n=32$ cells; $p<0.01$, $t$ test) (Fig. $1 B$ ). The cumulative probability of mEPSC amplitude shifted significantly to the right in SD rats compared with controls ( $p<0.01$, Kolmogorov-Smirnov test) (Fig. $1 B$ ). The mean amplitude of mEPSCs was also larger in SD rats than in controls (SD rats: $23.77 \pm 1.03 \mathrm{pA}, n=29$ cells; controls: $20.30 \pm 0.73 \mathrm{pA}$, $n=32$ cells; $p<0.01, t$ test). Thus, synaptic strength in rat cortical neurons increases with sustained wake at both presynaptic and postsynaptic sites, independent of time of day.

In a third experiment, we compared mEPSCs recorded from a group of rats kept awake for $4 \mathrm{~h}(n=4)$ and those from another group in which SD was followed by $2 \mathrm{~h}$ of RS $(n=4)$. Sleep quantity and quality during the recovery period were monitored by using standard electroencephalogram recordings as in previous reports (Vyazovskiy et al., 2008). RS rats spent most of the $2 \mathrm{~h}$ recovery period asleep (percentage of total recording time: nonrapid eye movement sleep, $72.4 \pm 3.9 \%$; rapid eye movement sleep, $13.1 \pm 0.6 \%)$. In these animals, the frequency of mEPSCs was significantly lower than in SD rats (SD rats: $2.44 \pm 0.48 \mathrm{~Hz}$, $n=10$ cells; RS rats: $1.02 \pm 0.16, n=9$ cells; $p<0.05$, $t$ test) (Fig. $1 C)$. The cumulative probability of mEPSC amplitude recorded from RS rats shifted significantly to the left compared with the SD group ( $p<0.01$, Kolmogorov-Smirnov test) (Fig. $1 C$ ), and the mean amplitude of mEPSCs was also significantly smaller (SD rats: $30.81 \pm 2.36 \mathrm{pA}, n=10$ cells; RS rats: $21.23 \pm 0.60 \mathrm{pA}, n=$ 9 cells; $p<0.01, t$ test). Thus, these results show that net synaptic strength of layer II/III pyramidal neurons in rat frontal cortex decreases with recovery sleep.

Finally, we tested whether sleep/wake can affect synaptic strength in a different animal species. To this end, coronal cortical slices $(300 \mu \mathrm{m})$ were prepared from mice (C57BL/6 males, 3-4 weeks old) killed at the same circadian time after $4 \mathrm{~h}$ of either 

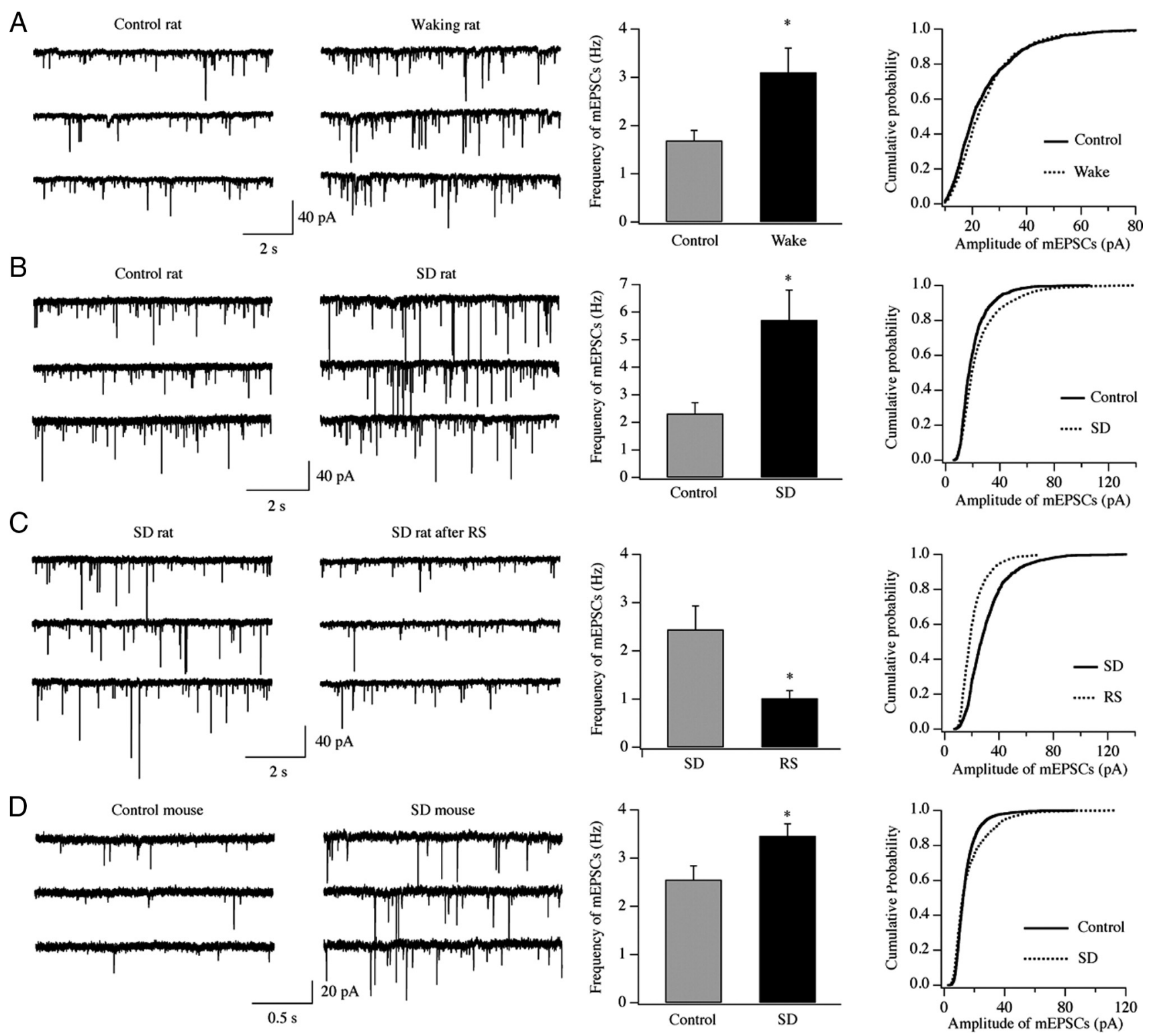

Figure 1. $\quad \boldsymbol{A}-\boldsymbol{D}$, Synaptic efficacy of glutamatergic synapses on layer II/III pyramidal neurons is enhanced during spontaneous and forced wakefulness in the frontal cortex of rats $(\boldsymbol{A}-\boldsymbol{C})$ and $\mathrm{mice}$ (D). Left, Representative traces of $\mathrm{mEPSC}$. Middle, Changes in the frequency of $\mathrm{mEPSCS}\left({ }^{*} p<0.05, t\right.$ test). Right, cumulative probability of mEPSC amplitude $(p<0.01$, Kolmogorov-Smirnov test). The number of animals is as follows: $\boldsymbol{A}, n=4$ rats/group (controls, 24 cells; wake, 25 cells); $\boldsymbol{B}, n=7$ rats/group (controls, 32 cells; SD rats, 29 cells); $\boldsymbol{C}, n=4$ rats $/$ group (SD rats, 10 cells; RS rats, 9 cells); $\boldsymbol{D}, n=4$ mice/group (controls, 24 cells; SD rats, 19 cells). To generate cumulative probability curve, 1000 to 2000 events from all recorded neurons in each group were used (A: controls, 1200 events; wake, 1250 events; $\boldsymbol{B}$ : controls, 1576 events, SD rats, 1441 events; $\boldsymbol{C}:$ SD rats, 997 events; RS rats, 900 events; $\boldsymbol{D}$ : controls, 3729 events; SD rats, 3856 events). Note that rats in $\boldsymbol{B}$ have a higher frequency of mEPSCs relative to those in $\boldsymbol{A}$ and $\boldsymbol{C}$, most likely due to slight changes in the experimental conditions used to prepare the slices.

SD by gentle handling (from 8:00 A.M. to 12:00 P.M., $n=4$ ) or of predominant sleep (controls, $n=4$ ). As shown in Figure $1 D$, the frequency of mEPSCs recorded after sustained waking was $3.45 \pm$ $0.24 \mathrm{~Hz}(n=19$ cells $)$, while it was $2.55 \pm 0.28 \mathrm{~Hz}(n=24$ cells $)$ after sleep ( $p<0.05, t$ test). Moreover, the cumulative probability curves for the amplitude of mEPSC events recorded from SD mice shifted significantly to the right relative to those recorded from controls ( $p<0.01$, Kolmogorov-Smirnov test) (Fig. 1D), indicating that the amplitude of mEPSCs had increased (mean mEPSC amplitude increased at trend level; SD rats: $13.43 \pm 1.58$ $\mathrm{pA}, n=19$ cells; controls: $11.94 \pm 0.71 \mathrm{pA}, n=24$ cells; $p>0.05$, $t$ test). Thus, these data suggest that in mice, as in rats, synaptic efficacy increases after sustained wake at both presynaptic and postsynaptic glutamatergic sites in layer II/III pyramidal neurons.

\section{Discussion}

The present findings provide the first direct demonstration that cortical synaptic efficacy increases after periods of wakefulness and is restored to lower levels after periods of sleep, independent of time of day. Synaptic strength was assessed directly by measuring mEPSCs in slices of frontal cortex of both mice and rats. Since both frequency and amplitude of mEPSCs increased after wake and decreased after sleep, it appears that changes in synaptic strength across wakefulness and sleep occurred both presynaptically and postsynaptically, and in the same direction. It is noteworthy that the significant increase in MEPSC amplitude in the spontaneously awake group relative to the sleeping group was not as large as the increase in SD rats relative to their controls (or RS rats). This is not surprising and likely reflects the fact that (1) 
wakefulness was more consolidated in animals forced to stay awake than in spontaneously awake animals, and (2) SD animals were awake longer than spontaneously awake animals (SD was enforced for $4 \mathrm{~h}$ starting at light onset, i.e., after the dark period, when rodents are mostly awake).

This direct demonstration of synaptic homeostasis across wakefulness and sleep is supported by previous molecular evidence showing an increase in AMPA receptor density in synaptoneurosomes, and related changes in protein phosphorylation, after wake and a decrease after sleep (Vyazovskiy et al., 2008). Moreover, the changes in mEPSCs observed in slices are consistent with the report that the slope and amplitude of field potentials increase in wake and decrease in sleep in freely moving rats (Vyazovskiy et al., 2008), and with similar observations in humans using transcranial magnetic stimulation and high-density EEG (Bellina et al., 2008). Recent anatomical evidence from Drosophila also supports a homeostatic regulation of synaptic strength (Donlea et al., 2009; Gilestro et al., 2009). Specifically, overall protein levels of both presynaptic and postsynaptic components of central synapses in the fruit fly were higher after waking and lower after sleep. These changes were related to behavioral state rather than time of day and occurred in all major areas of the Drosophila brain. Moreover, the decrease of synaptic markers during sleep was progressive, and sleep appeared necessary for their decline (Gilestro et al., 2009). Another study in Drosophila also found morphological evidence, in a specific neural circuit (circadian PDF neurons), for synaptic growth after sleep deprivation (Donlea et al., 2009).

It remains to be seen whether a homeostatic alternation of potentiation and depression of synaptic efficacy with the sleep/ wake cycle also occurs in other cortical areas and in noncortical structures. Intriguingly, a recent study found that the wakepromoting agent modafinil, as well as sleep deprivation, potentiated glutamatergic synapses on hypocretin/orexin neurons in the lateral hypothalamus (Rao et al., 2007), suggesting that changes in synaptic efficacy during the sleep/wake cycle could be widespread also in mammals. Moreover, it remains to be determined whether net changes in strength during sleep and wake occur in the adult brain as well as during early developmental periods characterized by high neuronal plasticity, extensive synaptogenesis, and massive synaptic pruning (Feinberg, 1982; Cowan et al., 1984; Carskadon et al., 2004; Campbell and Feinberg, 2009).

Finally, it is currently unknown whether net synaptic potentiation and depression across wake and sleep are activitydependent processes, similar to those involved in Hebbian and non-Hebbian synaptic plasticity (Turrigiano, 2008; Kessels and Malinow, 2009), and why these processes would not be balanced at all times within each behavioral state. Conceivably, during wakefulness there could be a bias toward potentiation because of the following: (1) animals interact with the environment and should form memories of important interactions; (2) neurons tend to signal important objects or events by firing strongly (over a low spontaneous firing rate), rather than little; (3) strong signals percolate best through the CNS if synapses between presynaptic and postsynaptic neurons are strengthened, rather than weakened; and (4) wakefulness is associated with high levels of neuromodulators such as acetylcholine and norepinephrine (Jones, 2005), which together appear to favor potentiation (Cirelli et al., 1996; Cirelli and Tononi, 2000; Seol et al., 2007). Sleep, on the other hand, and in particular slow-wave sleep, would favor global synaptic depression because of the following: (1) animals do not interact with the environment and therefore should not form new memories; (2) neurons undergo repeated sequences of de- polarization/synchronous firing (up-phase) and hyperpolarization/silence (down-phase) at $\sim 1 \mathrm{~Hz}$ (Steriade and Timofeev, 2003), which may be conducive to synaptic depression (Kemp and Bashir, 2001; Werk and Chapman, 2003; Birtoli and Ulrich, 2004; Rosanova and Ulrich, 2005; Werk et al., 2006; Czarnecki et al., 2007; Lubenov and Siapas, 2008); (3) the low level of neuromodulators such as acetylcholine, noradrenaline, serotonin, hypocretin, and histamine (Jones, 2005); and (4) a reduced expression of BDNF, whose presence prevents synaptic depression (Jiang et al., 2003).

Whatever the specific mechanisms leading to a net synaptic potentiation after wakefulness, and to a net synaptic depression after sleep, the present results lend support to the idea that a progressive synaptic imbalance may be a price the brain pays for learning, and that sleep may play an important role in restoring synaptic strength to a level that is energetically sustainable and permits further learning (Tononi and Cirelli, 2006).

\section{References}

Attwell D, Laughlin SB (2001) An energy budget for signaling in the grey matter of the brain. J Cereb Blood Flow Metab 21:1133-1145.

Bellina V, Huber R, Rosanova M, Mariotti M, Tononi G, Massimini M (2008) Cortical excitability and sleep homeostasis in humans: a TMS/hdEEG study. J Sleep Res 17 [Suppl 1]:39.

Birtoli B, Ulrich D (2004) Firing mode-dependent synaptic plasticity in rat neocortical pyramidal neurons. J Neurosci 24:4935-4940.

Campbell IG, Feinberg I (2009) Longitudinal trajectories of non-rapid eye movement delta and theta EEG as indicators of adolescent brain maturation. Proc Natl Acad Sci U S A 106:5177-5180.

Carskadon MA, Acebo C, Jenni OG (2004) Regulation of adolescent sleep: implications for behavior. Ann N Y Acad Sci 1021:276-291.

Cirelli C (2009) The genetic and molecular regulation of sleep: from fruit flies to humans. Nat Rev Neurosci 10:549-560.

Cirelli C, Tononi G (2000) Differential expression of plasticity-related genes in waking and sleep and their regulation by the noradrenergic system. J Neurosci 20:9187-9194.

Cirelli C, Tononi G (2008) Is sleep essential? PLoS Biol 6:e216.

Cirelli C, Pompeiano M, Tononi G (1996) Neuronal gene expression in the waking state: a role for the locus coeruleus. Science 274:1211-1215.

Cirelli C, Gutierrez CM, Tononi G (2004) Extensive and divergent effects of sleep and wakefulness on brain gene expression. Neuron 41:35-43.

Collingridge GL, Isaac JT, Wang YT (2004) Receptor trafficking and synaptic plasticity. Nat Rev Neurosci 5:952-962.

Cowan WM, Fawcett JW, O’Leary DD, Stanfield BB (1984) Regressive events in neurogenesis. Science 225:1258-1265.

Czarnecki A, Birtoli B, Ulrich D (2007) Cellular mechanisms of burst firingmediated long-term depression in rat neocortical pyramidal cells. J Physiol 578:471-479.

Donlea JM, Ramanan N, Shaw PJ (2009) Use-dependent plasticity in clock neurons regulates sleep need in Drosophila. Science 324:105-108.

Feinberg I (1982) Schizophrenia: caused by a fault in programmed synaptic elimination during adolescence? J Psychiatr Res 17:319-334.

Gilestro GF, Tononi G, Cirelli C (2009) Widespread changes in synaptic markers as a function of sleep and wakefulness in Drosophila. Science 324:109-112.

Horne J (1988) Why we sleep: the functions of sleep in humans and other mammals. Oxford: Oxford UP.

Jiang B, Akaneya Y, Hata Y, Tsumoto T (2003) Long-term depression is not induced by low-frequency stimulation in rat visual cortex in vivo: a possible preventing role of endogenous brain-derived neurotrophic factor. J Neurosci 23:3761-3770.

Jones BE (2005) From waking to sleeping: neuronal and chemical substrates. Trends Pharmacol Sci 26:578-586.

Kemp N, Bashir ZI (2001) Long-term depression: a cascade of induction and expression mechanisms. Prog Neurobiol 65:339-365.

Kessels HW, Malinow R (2009) Synaptic AMPA receptor plasticity and behavior. Neuron 61:340-350.

Krueger JM, Rector DM, Roy S, Van Dongen HP, Belenky G, Panksepp J (2008) Sleep as a fundamental property of neuronal assemblies. Nat Rev Neurosci 9:910-919. 
Lubenov EV, Siapas AG (2008) Decoupling through synchrony in neuronal circuits with propagation delays. Neuron 58:118-131.

Malenka RC, Bear MF (2004) LTP and LTD: an embarrassment of riches. Neuron 44:5-21.

Moruzzi G (1966) The functional significance of sleep with particular regard to the brain mechanisms underlying consciousness. In: Brain and conscious experience (Eccles JC, ed), pp 345-388. New York: Springer.

Rao Y, Liu ZW, Borok E, Rabenstein RL, Shanabrough M, Lu M, Picciotto MR, Horvath TL, Gao XB (2007) Prolonged wakefulness induces experience-dependent synaptic plasticity in mouse hypocretin/orexin neurons. J Clin Invest 117:4022-4033.

Rosanova M, Ulrich D (2005) Pattern-specific associative long-term potentiation induced by a sleep spindle-related spike train. J Neurosci 25: 9398-9405.

Seol GH, Ziburkus J, Huang S, Song L, Kim IT, Takamiya K, Huganir RL, Lee HK, Kirkwood A (2007) Neuromodulators control the polarity of spiketiming-dependent synaptic plasticity. Neuron 55:919-929.

Siegel JM (2009) Sleep viewed as a state of adaptive inactivity. Nat Rev Neurosci 10:747-753.
Steriade M, Timofeev I (2003) Neuronal plasticity in thalamocortical networks during sleep and waking oscillations. Neuron 37:563-576.

Tononi G, Cirelli C (2006) Sleep function and synaptic homeostasis. Sleep Med Rev 10:49-62.

Turrigiano GG (2008) The self-tuning neuron: synaptic scaling of excitatory synapses. Cell 135:422-435.

Ungless MA, Whistler JL, Malenka RC, Bonci A (2001) Single cocaine exposure in vivo induces long-term potentiation in dopamine neurons. Nature 411:583-587.

Vyazovskiy VV, Cirelli C, Pfister-Genskow M, Faraguna U, Tononi G (2008) Molecular and electrophysiological evidence for net synaptic potentiation in wake and depression in sleep. Nat Neurosci 11:200-208.

Vyazovskiy VV, Olcese U, Lazimy YM, Faraguna U, Esser SK, Williams JC, Cirelli C, Tononi G (2009) Cortical firing and sleep homeostasis. Neuron 63:865-878.

Werk CM, Chapman CA (2003) Long-term potentiation of polysynaptic responses in layer $\mathrm{V}$ of the sensorimotor cortex induced by thetapatterned tetanization in the awake rat. Cereb Cortex 13:500-507.

Werk CM, Klein HS, Nesbitt CE, Chapman CA (2006) Long-term depression in the sensorimotor cortex induced by repeated delivery of $10 \mathrm{~Hz}$ trains in vivo. Neuroscience 140:13-20. 